\section{Severe cold lower limbs in patients with Parkinson's disease during the summer}

\author{
Hiroshi Kataoka, Satoshi Ueno \\ Department of Neurology, Nara Medical \\ University, Kashihara, Nara, Japan
}

\section{Abstract}

Parkinson's disease (PD) is frequently associated with vasomotor symptoms such as distal cold limbs or sensitivity to cold. Coldness of the lower limbs (COL) usually occurs in winter and is often accompanied by pain, potentially causing difficulty in walking or standing. A standard dopaminergic treatment for such symptoms is yet to be established. We describe two patients with $\mathrm{PD}$, who had severe COL during summer. For example, the patients wore many pairs of socks or used heating appliances in the summer. Severe COL can occur in summertime and can be intolerable or unpleasant, since it can worsen disability. The treatment with a dopamine agonist did not sufficiently decrease the severity of $\mathrm{COL}$.

\section{Introduction}

Parkinson's disease (PD) is frequently associated with vasomotor symptoms such as distal cold limbs or sensitivity to cold, estimated to occur in $40-50 \%$ of patients. ${ }^{1}$ Cold limbs usually occur in the winter and are often accompanied by pain, potentially causing difficulty in walking or standing. A standard dopaminergic treatment for such symptoms has yet to be established. We describe two patients with PD who had severe coldness of the lower limbs (COL) in the summer. For example, patients wore many pairs of socks or used heating appliances in the summer.

\section{Case Report \#1}

A 75-year-old man noticed slowness and right-hand tremor in 2010. Bradykinesia, rightdominant rigidity in all four limbs, and righthand resting tremor developed in October 2010, and these parkinsonian features responded to treatment with levodopa. In October 2011, he presented with painful numbness in his legs, and walking became difficult. The dose of levodopa was gradually increased to $500 \mathrm{mg} /$ day, and treatment with selegiline $(7.5 \mathrm{mg} /$ day $)$ was started subsequently. The symptoms decreased.
The patient had received levodopa and selegiline in stable doses for 17 months. In September 2013, he had visual hallucinations of insects, and in December he showed wearing-off with painful muscle tightness in the lower limbs. The muscle tightness was present at rest and when moving his legs, and he shouted out it hurts. The score on the unified Parkinson's Disease Rating Scale (UPDRS), part III was 63 . He could not stand or walk. Zonisamide was initiated ( $25 \mathrm{mg} /$ day), and in January 2014 the dose was increased to 50 $\mathrm{mg}$ /day. In early April, the severity of both the muscle tightness and pain decreased, and he became able to walk alone. The score on UPDRS, part III had decreased to 32 . In late April, the painful tightness recurred, especially in the evening, and he could walk only in the morning. Rotigotine $(4.5 \mathrm{mg} /$ day $)$ was started in addition to levodopa (500 mg/day), selegiline ( $7.5 \mathrm{mg} /$ day), and zonisamide ( $50 \mathrm{mg} /$ day). The dose of rotigotine was increased to $9 \mathrm{mg}$ /day in May and to $18 \mathrm{mg} /$ day in June. The off-periods were markedly reduced to 2 hours per day. In the summer of 2014, his family noticed that everyday he wore overlapping multiple shirts, used electric blankets, or switched on the floor heating, which was not tolerable by his family, because he felt that his legs were too cold and painful. When the COL was accompanied by pain, he could not stand, walk, or turn over during sleep. In late August, we asked the patient whether the severity of the painful COL had changed after increasing the dosage of rotigotine, and he responded that the severity had decreased and that warming his legs was more effective. However, painful coldness was evident after the evening. At this time, the score on UPDRS, part III was 21.

\section{Case Report \#2}

In 2004, an 80-year-old woman had bradykinesia, resting hand tremor, and rigidity of the right limbs, which responded to treatment with levodopa. In January 2009, wearing-off developed, and pergolide ( $500 \mu \mathrm{g} /$ day) was started. In January 2011, she noticed tightness and pain in her upper limbs. In April, the patient was given levodopa $(500 \mathrm{mg} /$ day) and pergolide (500 $\mu \mathrm{g} /$ day), but off-periods were prolonged, and treatment with entacapone was begun in October. Subsequently, the dose was increased to $600 \mathrm{mg} /$ day. In March 2012, the tightness and pain extended to her legs, and muscle cramps were often present in her legs. In April 2012, she noticed COL and tightness persisting for several hours before taking antiparkinsonian medications. The score on the MMSE was 27. She was given levodopa (300 mg/day), entacapone $(600 \mathrm{mg} /$ day $)$, and pergolide (500 $\mu \mathrm{g} /$ day). Ropinirole was started, and the dose of
Correspondence: Hiroshi Kataoka, Department of Neurology, Nara Medical University, 840 Shijocho, Kashihara, Nara 634-8522, Japan. Tel.: +81.744.29.8860 - Fax: +81.744.24.6065. E-mail: hk55@naramed-u.ac.jp

Key words: Parkinson; Cold limbs; Vasomotor symptoms; Autonomic dysfunction; Dopamine agonist; Dopaminergic medications.

Contributions: the authors contributed equally.

Conflict of interest: the authors declare no potential conflict of interest.

Received for publication: 2 July 2016

Revision received: 15 August 2016.

Accepted for publication: 25 August 2016.

This work is licensed under a Creative Commons Attribution NonCommercial 4.0 License (CC BYNC 4.0).

(C) Copyright H. Kataoka and S. Ueno, 2016 Licensee PAGEPress, Italy

Neurology International 2016; 8:6676

doi:10.4081/ni.2016.6676

pergolide was increased to $750 \mu \mathrm{g} / \mathrm{day}$. In February 2014, the dose of extended-release ropinirole was increased ( $8 \mathrm{mg} /$ day). The severity of the painful tightness mildly decreased, but the coldness was unchanged. In early April, rotigotine ( $4.5 \mathrm{mg} /$ day) was begun because the patient had difficulty walking since severe COL had developed, leading to prolonged off-periods. In late April, she still wore many pairs of socks and put hot packs into the socks. These actions continued during the summer. In July, the dose of rotigotine was increased to $18 \mathrm{mg} /$ day. In August, the pain disappeared, and the off-periods due to COL became slightly shorter. However, COL persisted.

\section{Characteristics of the cold limbs}

COL occurred every day and in the summer. The coldness caused difficulty in waking. To avoid coldness, patients warmed their limbs in the summer by actions that were usually not done in the summer, such as using heating appliances. The patients had painful muscle tightness before the onset of COL. The coldness was often accompanied by such tightness. All patients had other autonomic symptoms, such as constipation. Zonisamide or entacapone was initiated or the dose was increased within 12 months before $\mathrm{COL}$, but the duration of COL was prolonged. The coldness was likely to mildly respond to dopamine agonists, but not enough to satisfy these patients. In the winter of the year when patients had COL in the summer, both patients took actions similar to those in the summer to avoid coldness. However, the former patient did not complain 
Table 1. Clinical characteristics of two patients with Parkinson's disease who had severe coldness of the lower limbs.

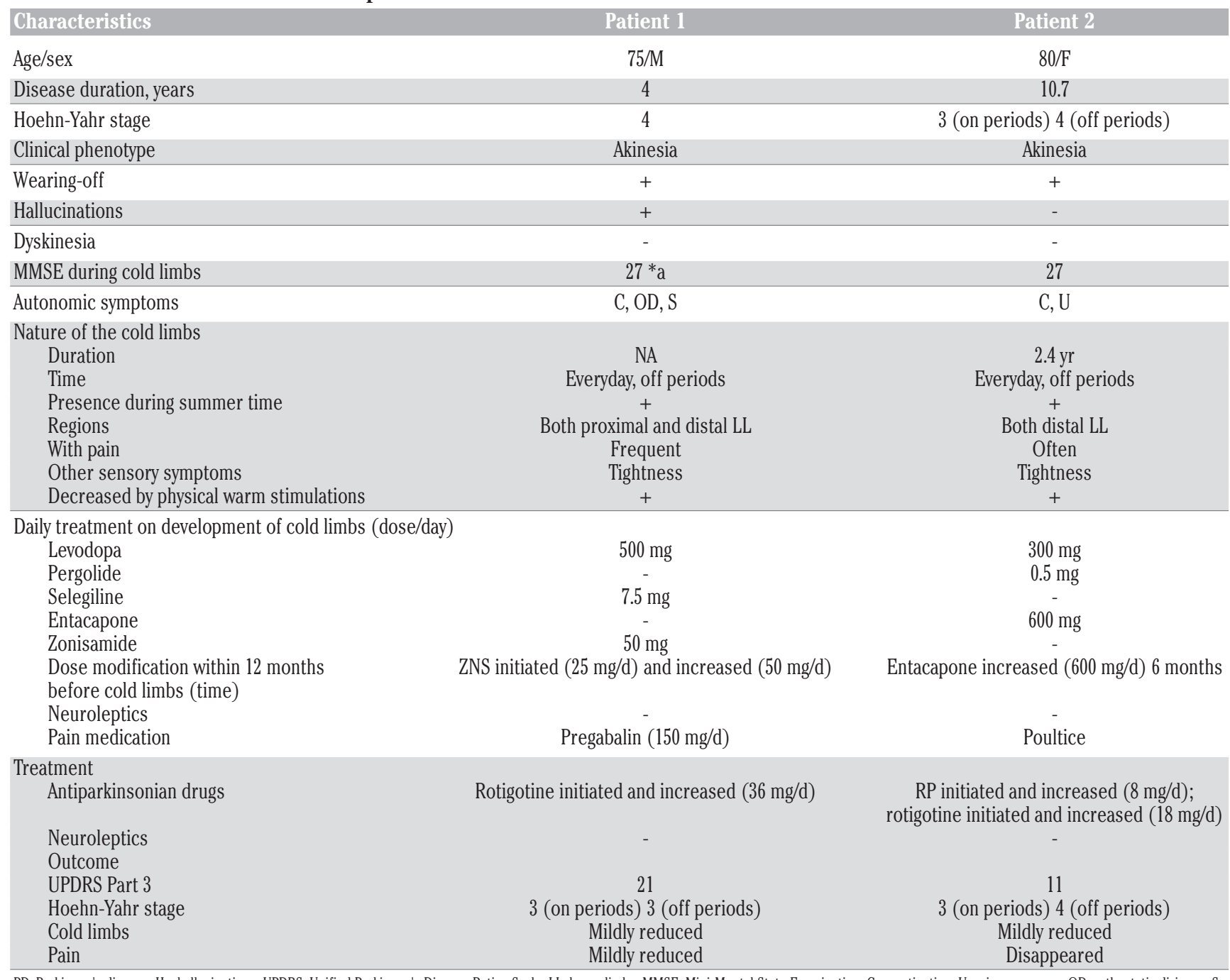

PD, Parkinson's disease; Hs, hallucinations; UPDRS, Unified Parkinson's Disease Rating Scale; LL, lower limbs; MMSE, Mini-Mental State Examination; C, constipation; U, urinary urgency; OD, orthostatic diziness; S, sweating abnormalities; m, months; d, day; ND, not done. *a: examinated in September 2014 when cold limbs were present.

much about COL in the summer. The latter patient complained more frequently about COL.

\section{Discussion and Conclusions}

Information on the interval between the start of anti-parkinsonian treatment and the onset of COL was not provided in previous reports on patients with PD. ${ }^{2}$ The intervals were long in our patients. Long-term dopaminergic treatment may not directly elicit, but might be prerequisite to the development of COL, as well as psychosis or hallucinations. ${ }^{3}$ Central autonomic or peripheral autonomic impairment has been suggested to participate in the mechanism of vasomotor abnormalities such as distal COL in PD. ${ }^{2,4}$ Recently, one study assessing skin sympathetic nerve activity in PD patients with COL showed that the skin blood circulation was decreased by prolonged vasoconstriction, and denervation hypersensitivity of adrenergic receptors in cutaneous blood vessels might lead to vasoconstriction. ${ }^{2} \mathrm{~A}$ histological study of skin specimens in patients with PD demonstrated decreased autonomic innervation of cutaneous vessels. ${ }^{5}$ Thermoregulatory central autonomic failure has been shown to be caused by lesions in the hypothalamus. ${ }^{6}$ Such peripheral and central autonomic impairment may be major causative factors for COL. In our patients, $\mathrm{COL}$ was preceded by painful muscle tightness. PD is associated with a reduction in muscle sympathetic nerve activity controlling blood pressure. ${ }^{7}$ Reduced sympathetic nerve activity associated with muscle tightness might also attribute to $\mathrm{COL}$.

In a previous study, 8 of 13 patients with PD who had COL were treated with pergolide or an ergoline-based dopamine agonist, and there was no significant difference in measured data, including recovery time, between patients with and those without COL. ${ }^{2}$ However, detailed information was not reported. Similarly, dopamine agonists were used to treat COL in our patients, but their effectiveness was limited.

Severe COL can occur in summertime and can be intolerable or unpleasant; COL can worsen disability. Treatment with a dopamine agonist did not sufficiently decrease the severity of COL. In Table 1 are reported the characteristics of the two patients with Parkinson's disease who had severe coldness of the lower limbs.

\section{References}

1. Witjas T, Kaphan E, Azulay JP, et al. onmotor fluctuations in Parkinson's disease: frequent and disabling. Neurology 
2002;59:408-13.

2. Shindo K, Kobayashi F, Miwa M, et al. Temporal prolongation of decreased skin blood flow causes cold limbs in Parkinson's disease. J Neural Transm 2013;120:445-51.

3. Moskowvitz C, Moses H, Klawans HL. Levodopa-induced psychosis: a kindling phenomenon. Am J Psychiatry 1978;135:669-74.
4. Suchowersky 0, Reich S, Perlmutter J, et al. Practice parameter: diagnosis and prognosis of new onset Parkinson disease (an evidence-based review): report of the Quality Standards Subcommittee of the American Academy of Neurology. Neurology 2006;66:968-75.

5. Dabby R, Djaldetti R, Shahmurov M, et al. Skin biopsy for assessment of autonomic denervation in Parkinson's disease. J
Neural Transm 2006;113:1169-76.

6. De Marinis M, Stocchi F, Testa SR, et al. Alterations of thermoregulation in Parkinson's disease. Funct Neurol 1991;6:279-83.

7. Shindo K, Watanabe H, Tanaka H, et al. Age and duration related changes in muscle sympathetic nerve activity in Parkinson's disease. J Neurol Neurosurg Psychiatry 2003;74:1407-11. 